\title{
Naevus, naevus pigmentoso e melano-sarcoma
}

\author{
Pelo Prof. Dr. Walther Haberfeld, \\ Cathedratico de Anatomia-Pathología da \\ Faculdade de Medicina e Cirurgia de S. Paulo.
}

Um grande numero de dermatologistas inclúe sob a denominação de "naevus" muitos tumores differentes, pequenos e grandes, assim por ex.: as keratodermias, os tumores cheloides, etc.

Antigamente chamava-se "naevus" toda anomalia cutanea circumscripta que se distinguisse pela côr e, pela sua permanencia durante toda a vida, se apresentasse quasi como um signo individual. Essa definição é valida ainda hoje.

A melhor definição de naevus foi dada por Unna: anomalias cutaneas circumscriptas e pequenas, de disposição hereditaria ou de formação embryonaria, as quaes apparecem em qualquer época da vida, possuem um desenvolvimento muito lento e se distinguem pela sua côr e fórma.

As anomalias cutaneas circumscriptas, pigmentosas ou fibrosas, de volume grande, pertencem anatomicamente ao mesmo grupo, e são, por isso, denominadas naevi gigantes.

Não pertence ao nosso assumpto discutir a justificação das diversas subdivisões do naevus, assumpto esse que faz parte da dermatologia. Queremos, apenas, salientar que, logicamente, abstracção feita do naevus vasculoso, cuja denominação melhor seria a de "hemangioma", deveriamos chamar de naevus apenas os tumores cutaneos que se caracterisam pela presença das tão discutidas cellulas de naevus. 
Essas cellulas de naevus se reunem sempre em grupos ou em ilhotas, separadas por feixes de tecido conjunctivo. A fórma dessas ilhotas é oval ou alongada, ás vezes muito alongada ,e na base do naevus as ilhotas são menores e mais unidas, afastando-se para a superficie cutanea (isto é, para cima) em fórma de leque (não existe ahi a contra-pressão das fibras elasticas da sub-cutis; por esse motivo, dá-se tambem a saliencia na superficie).

Entre essas ilhotas e as cellulas epidermicas existe quasi sempre tecido conjunctivo, isto é, raramente as cellulas de naevus chegam até o contacto immediato com a epiderme. Essas cellulas são de tamanho medio, menores do que as cellulas epidermicas, de fórma redonda-oval, com nucleo relativamente grande e vesiculoso, podem ter ou não pigmento melanico.

A proveniencia das cellulas de naevus é até hoje uma questão não resolvida. Recklinghausen e depois delle muitos outros as consideram como provenientes de endothelios dos vasos lymphaticos, chamando os naevus de lymphangiofibroma. Recklinghausen quasi só dava provas negativas, excluindo da formação do naevus os nervos, as glandulas sudoriparas, arterias, veias, etc. As ilhotas nascem, dizem os autores, por proliferação dos endothelios lymphaticos até a occlusão da luz do vaso. O endothelio lymphatico que reveste a peripheria das ilhotas prova que se trata de vasos lymphaticos (Borst).

E, com effeito, em certos naevus, é possivel ver uma formação analoga. (Vide fig. I).

Uma segunda opinião consiste em que as cellulas de naevus sejam de origem epithelial, respectivamente epidermal, opinião aventada e defendida por Unna. Baseia-se sobre os seguintes factos: podemos observar nas manchas pigmentosas das crianças (estas são de estructura differente das do adulto) uma proliferação de cellulas epidermicas com invasão da cutis. Estas cellulas entram depois num processo de metaplasia regressiva, perdendo a propriedade de producção de fibras protoplasmaticas, tão caracteristicas para as cellulas epidermicas. Aquellas cellulas proliferadas têm tendencia a se separar da "matrix" (pelo chamado processo de Abtropfen - cahir em fórma de gotta), ficando 


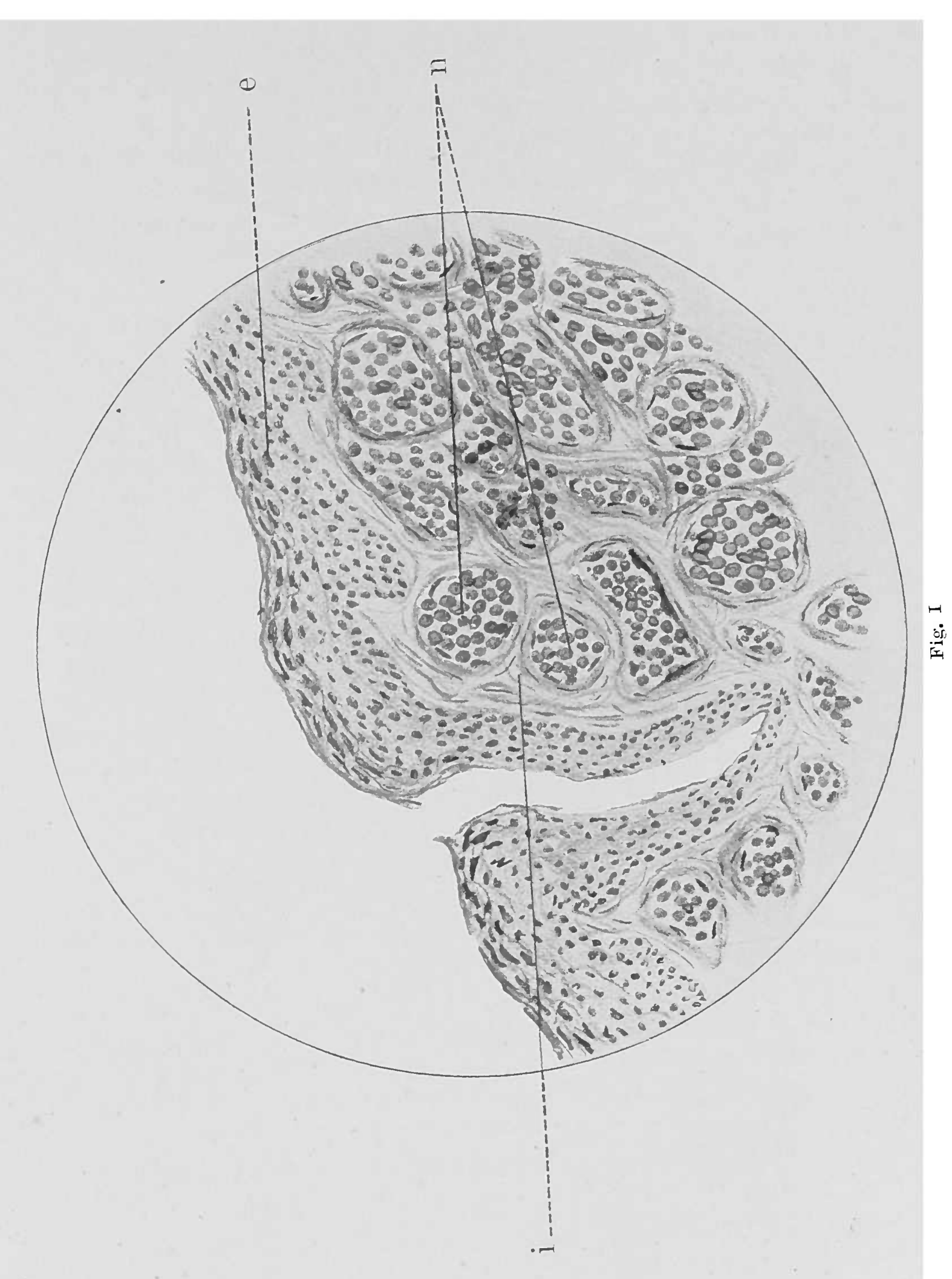



envolvidas por tecido conjunctivo e por este separadas em ilhotas. Conservam, porém, todos os signaes de sua origem epithelial: nucleo vesiculoso, ausencia completa de qualquer substancia intercellular. (Vide fig. II)

Ribbert, que, no principio, era partidario da opinião de Recklinghausen, formou nova explicação sobre a formação das cellulas de naevus: sua origem chromatophorica, isto é, de natureza conjunctiva, apoiando-se no facto de existir uma substancia intercellular fibrillar entre as cellulas, que assim ficam circumdadas, cada uma, por uma rêde de fibrillas. Elle considera essas cellulas de naevus como pre-phase não differenciada dos chromatophoros; por esta mesma razão não apresentam necessariamente a ramificação caracteristica dos chromatophoros nem seu pigmento melanico. Estas cellulas podem ser e deixar de ser pigmentadas. Uma outra prova da relação acima mencionada é o facto de se poder observar chromatophoros typicos situados entre o naevus e a epiderme, que penetram com seus prolongamentos entre as cellulas de naevus, e, ainda mais, ás vezes, demonstram uma transição directa para com as cellulas de naevus.

Como em todas as questões controversas da medicina, acontece tambem aqui que quanto mais aprofundado se torna o estudo do assumpto discutido, tanto menos fica simplificado, como se poderia pensar a priori; ao contrario, apresentam-se mais cheias de pontos duvidosos. Se devéras pudessemos observar em todos os naevi aquella substancia inter-cellular fibrillar de Ribbert, então, a origem conjunctiva do naevus estaria resolvida; porque não seria logico concluir com os partidarios de Unna que esta rêde fibrillar fosse o resto do tecido conjunctivo preexistente. Estes restos poderiam ser apenas fasciculos grossos, mas, nunca fibrillas finas rodeando as cellulas. Ainda menos se justifica a opinião de Krompecher, partidario de Unna, de que esta rêde fibrillar deveria ser explicada por uma metaplasia das cellulas epidermicas em cellulas conjunctivas. De outro lado, porém, ha muitos autores que negam decididamente a existencia verdadeira da' rêde fibrillar, autores estes que pretendem ter seguido rigorosamente a technica de Ribbert. Ribbert, por sua vez, explica o processo de Abtropfen de Unna como erro de interpretação, isto é, não como transição de 
cellulas epidermicas para as cellulas de naevus, mas, julga e demonstra sua these por figuras, dizendo que, mesmo em caso de transição apparente entre as cellulas mencionadas, estas duas cellulas sempre têm differenças nitidas e nunca apresentam uma transição verdadeira e, ainda mais, que estas cellulas de naevus já se caracterisaram antes como chromatophoros ou congeneres. (Vide fig. III).

A discussão sobre a genesis das cellulas de naevus só poderá ser definitivamente decidida, quando tivermos noções mais certas sobre a formação do pigmento melanico.

$\mathrm{Ha}$ bem pouco tempo, a formação deste pigmento melanico se ligou sómente aos chromatophoros, isto é, ás cellulas mesenchymaes; attribuiu-se, depois, tambem ás cellulas epitheliaes, respectivamente ectodermicas (cerebro!). Ultimamente, ha muitos autores que sustentam a these de que, unicamente as cellulas epidermicas sejam capazes da producção de pigmento e que os chromatophoros da pelle, por exemplo, retiram o pigmento das cellulas epidermicas, isto é, o possuem secundariamente sem o formar. Assim tambem, o pigmento na choroidea e na iris que, como é sabido, apresentam um numero enorme de chromatophoros, não deve ser formado nestas cellulas, mas, nas cellulas da retina, de origem ectodermica e dahi retirado pelos chromatophoros.

Como devemos então explicar a relação entre o naevus pigmentoso e melano-sarcoma clinica e anatomo-pathologicamente estabelecida?

O simples facto de que as cellulas do melano-sarcoma são tão differentes das do naevus e de que na dissecção apresentam fibrillas e prolongamentos similes aos dos chromatophoros, demonstra que a relação não póde ser directa; além disso, as cellulas do naevus e seus nucleos são muito menores do que as do melano-sarcoma. Outro facto ainda, em pequenos melano-sarcomas nascidos na visinhança de naevus pode-se observar o seguinte: o melano-sarcoma existe ao lado do naevus, desviando-o pela sua proliferação e podendo, ainda, penetrar entre as cellulas do naevus, (Verdade é que isto não fala em absoluto contra a origem de cellulas de naevus; pois que a neoformação podia provir só de uma parte das cellulas de naevus com compressão consecutiva das 


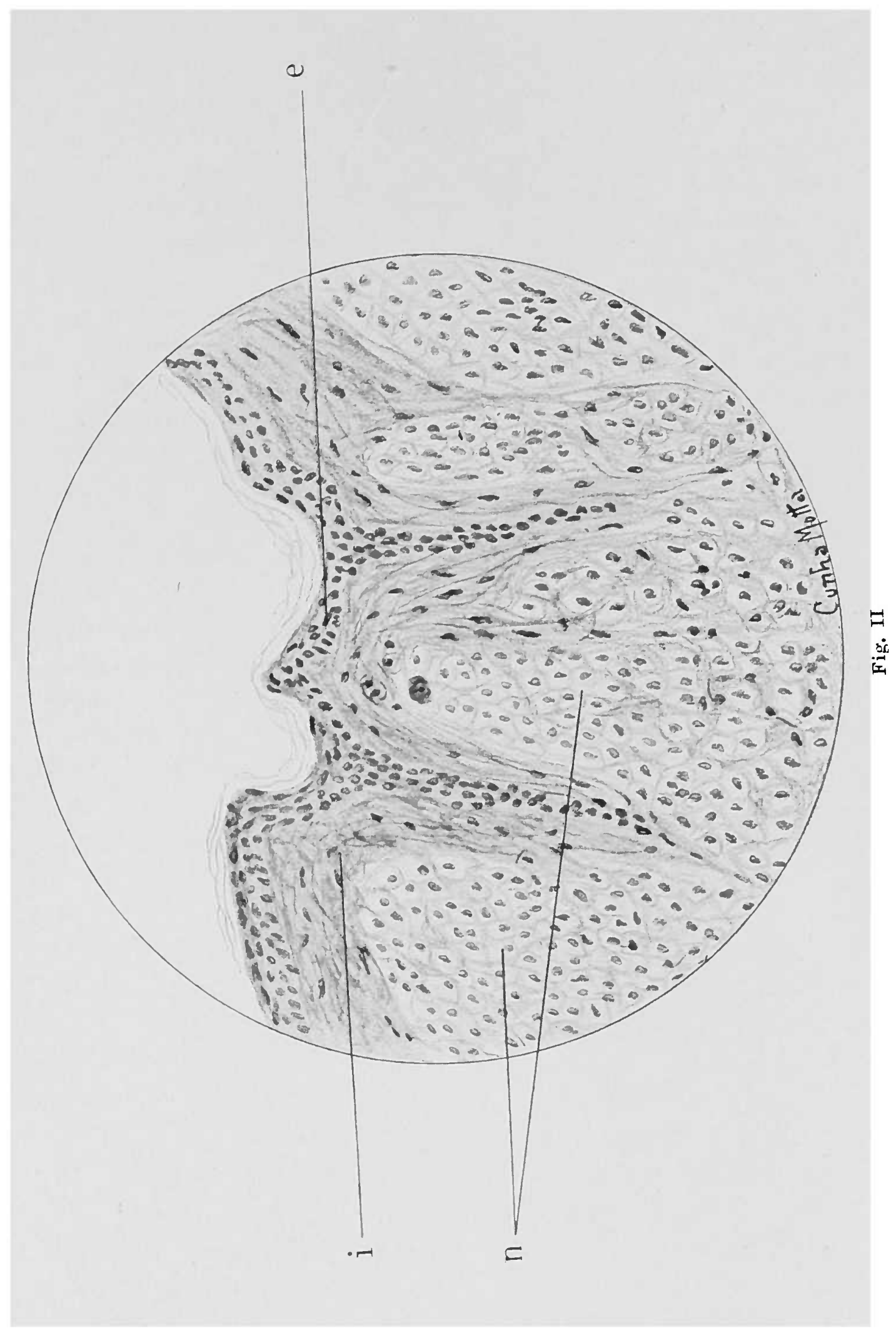





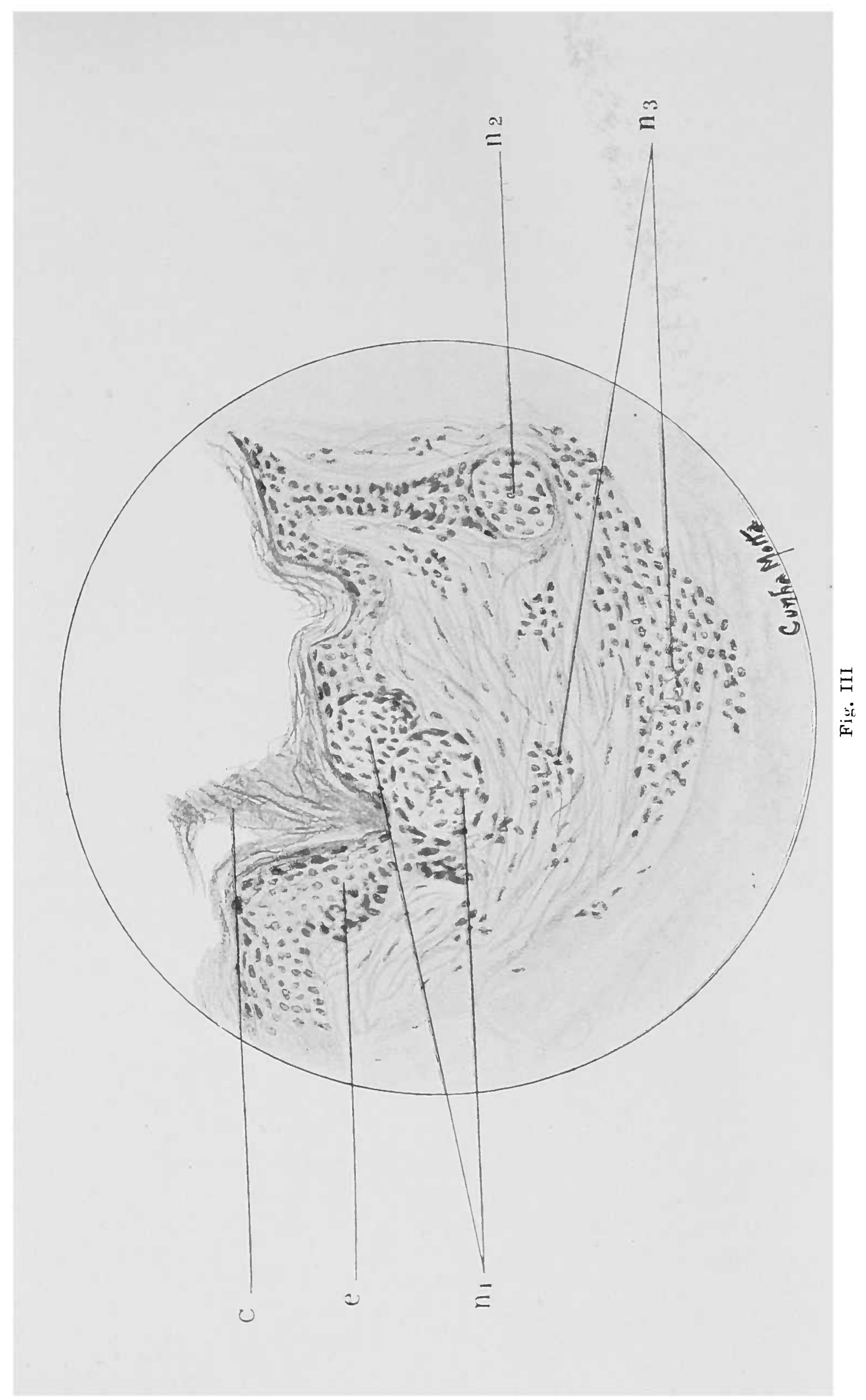



outras). Ha, tambem, melano-sarcomas, cujo desenvolvimento não se póde relacionar com cellulas de naevus, porque não existia anteriormente um naevus.

Podemos então dizer com certeza que as cellulas do melanoma se originam dos chromatophoros e não de cellulas de naevus. Comprehendemos facilmente que os melano-sarcomas se desenvolvem sobretudo em naevus pigmentosos que apresentam grande numero de cellulas chromatophoras. Ao principio se formam sómente melanomas, isto é, chromatophoromas pequenos, clinicamente benignos, que, mais tarde, talvez, sob a influencia de traumatismos communs ou therapeuticos se transformam em chromatophoromas malignos, chamados melano-sarcomas.

A relação unica entre o naevus e o melano-sarcoma consistiria, então, em que a presença anterior de um naevus ipso facto demonstrasse a presença de uma perturbação no tecido conjunctivo pigmentado, perturbação esta que mais tarde se póde manifestar pelo desenvolvimento de um melano-sarcoma. Naevus e melanomas têm então a mesma causa: perturbação no tecido conjunctivo pigmentado. Mas, não ha entre elles relação de causa e effeito.

N. B. - Caso ficasse provada a origem epithelial dos naevus, não se deveria, ao menos em certos tumores, falar de um melano-sarcoma, mas, de melano-carcinoma.

Explicação das figuras.

Figura I. Naevus não pigmentoso da pelle. Observa-se nitidamente a estructura alveolar como ás vezes tambem no melano-sarcoma, mas, ao mesmo tempo, faz lembrar um lymphangioma hypertrophico.

As cellulas de naevus ( $n$ ) encontram-se apparentemente dentro de uma luz preformada, revestida de cellulas chatas, dando a apparencia de cellulas endotheliaes. e=epiderme; $\mathbf{i}=$ tecido conjunctivo da cutis.

Fig. II. Naevus plano, com cellulas de naevus de apparencia epithelial (muito protoplasma, bem limitado, nucleo vesiculoso). Estas cellulas são separadas da epiderme (e) pelo tecido conjunctivo (i).

Fig III. Naevus pigmentoso. Aqui se encontram as cellulas de naevus (n. 1 e n. 2) em parte, em continuação directa com as cellu- 
las da epiderme, mas, apesar desta relação topographica intima, differenciam-se muito bem das cellulas epidermicas, formando ilhotas bem limitadas.

$\mathbf{n}_{1}=$ ilhotas de cellulas de naevus intraepidermicas, pigmentadas.

$\mathbf{n}_{2}=$ ilhotas intra-epidermicas, não pigmentadas.

$\mathbf{n}_{3}=$ cellulas de naevus espalhadas no tecido sub-epidermica.

$\mathbf{e}=$ epiderme com cellulas basaes pigmentadas.

$\mathbf{c}=$ istratum corneum.

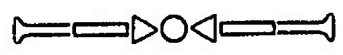 \\ 0 corpo calloso e as apraxías \\ Conferencía realísada no Centro Academico "0s- waldo Cruz", em 12-VIII-1916, pelo $40^{\circ}$ annista de Medícina, Joäo Procopio.}

\section{Meus senhores:}

Cabe-nos a immensa honra de inaugurar com este despretencioso e modesto trabalho, a série de conferencias de alumnos, em boa hora instituida pelo Centro Academico "Oswaldo Cruz".

Não fosse a intenção de collaborar com os dedicados collegas de Directoria, pondo em campo as pequenissimas e escassas forças de que dispomos, em pról do programma a que o "Centro" se propoz, não fosse o confiar na magnanimidade excessiva dos que vão ouvir a nossa palavra desconcertada e sem brilho, sem duvida haviamos de succumbir ao fardo que, braços vigorosos, mas, benevolentes, depuzeram sobre nossos hombros inexperientes e frageis.

A este trabalho, ainda que mostre a boa vontade de quem procurou fazel-o, falta por inteiro a originalidade; não vamos dizer cousas novas, não vamos trazer á luz da sciencia verdades por nós descobertas; o que procuramos realisar, é deixar aberta a estrada em que vós, meus distinctos collegas, haveis de jorrar as luzes do vosso incansavel esforço, das vossas pacientes observações, do vosso inces- 